Lexis Vol. XLI (2) 2017: 311-346

\title{
Estudio sociolingüístico del dequeísmo en el español hablado en Santiago de Chile"
}

\author{
Abelardo San Martín Núñez \\ Universidad de Chile
}

\begin{abstract}
RESUMEN
En este artículo se describe el empleo del dequeísmo en el habla santiaguina en la actualidad desde el punto de vista sociolingüístico. Para tal propósito, se analizó una muestra estratificada de entrevistas realizadas a 120 hablantes santiaguinos. Los objetivos del estudio consistieron en identificar los factores internos que propiciaban el empleo del dequeísmo y correlacionarlo con factores sociodemográficos del hablante (sexo, edad y grupo socioeconómico). Los resultados muestran que, en términos relativos, los contextos que más favorecen el dequeísmo son la sustitución de otras preposiciones por de y las estructuras apositivas, si bien la mayoría de los casos de dequeísmo se dieron en cláusulas con las funciones de objeto directo y de sujeto. Asimismo, los factores sociales más sensibles en la ocurrencia

\footnotetext{
* En este trabajo se sintetizan los resultados más relevantes de una de las secciones de nuestra tesis doctoral inédita, "Variación sintáctica y discursiva en el español hablado en Santiago de Chile. Análisis sociolingüístico del queísmo, el dequeísmo, el discurso referido y los marcadores de reformulación”, dirigida por el doctor Humberto López Morales y leída en la Universidad de Valladolid. Dedicamos este trabajo al Dr. Luis Prieto Vera en agradecimiento a su magisterio y como reconocimiento a su notable contribución al desarrollo de la sociolingüística en Chile.
} 
del dequeísmo fueron el sexo del informante y su edad. De este modo, se comprobó que el dequeísmo es más frecuente en los hombres y en los hablantes de edad avanzada de la muestra.

Palabras clave: español de Chile, sociolingüística, variación sintáctica, dequeísmo

\section{AbSTRACT}

Within a sociolinguistic framework, in this paper, we describe the use of dequeismo (use of 'de que' instead of 'que') in the utterances of Spanish speakers living in Santiago de Chile. A stratified sample of 120 interviews with people residing in Santiago was utilized for the analysis. The study intended to 1) identify the internal variables that prompted the use of dequeismo, and, 2) correlate those variables with sociodemographic of the speakers (gender, age, and socioeconomic status). The results suggest that the contexts that foster the use of dequeismo frequently allowed for the replacement of other de prepositions and appositive structures, although most cases occurred in clauses with functions of direct object and subject. Additionally, in the sample, the use of dequeismo was frequently coincided in the speeches of male and older speakers.

Keywords: Chilean Spanish, sociolinguistics, syntactic variation, dequeísmo

\section{Introducción}

En español, algunos hablantes vacilan en el empleo de la preposición $d e$, empleándola cuando no corresponde, según las reglas de la gramática española, en las cláusulas sustantivas o relativas introducidas por la conjunción o el pronombre que, fenómeno conocido como dequeísmo, ${ }^{1}$ lo que conlleva el correspondiente dictamen normativo o académico. ${ }^{2}$ El objetivo general de este trabajo — de carácter

\footnotetext{
1 Según Gómez Torrego (1999: 2107, nota 1), De Mello (1995) sugiere que fue Rabanales (1974) quien habría introducido el término "dequeísmo"; con anterioridad a su trabajo se caracterizaba a este fenómeno como "de expletiva ante que" o "uso superfluo de de ante que".

2 En el Esbozo de la gramática de la RAE (1973), el uso superfluo de la preposición de aparece escuetamente mencionado, al comentar algunos ejemplos en las
} 
cuantitativo y correlacional - es analizar el empleo del dequeísmo en una muestra de 120 entrevistas realizadas a informantes santiaguinos. Sus objetivos específicos son determinar cuáles son los factores lingüísticos que propician el uso de la variante dequeísta, en dicha muestra, y correlacionar las frecuencias de empleo de las variantes no dequeísta y dequeísta con las variables sociodemográficas: sexo, edad y grupo socioeconómico de los informantes. Nuestra hipótesis es que tanto los factores internos como los factores externos inciden en la ocurrencia del dequeísmo.

En este estudio consideramos el dequeísmo como un caso de variable sintáctica; por lo tanto, un problema a considerar es la extensión del ámbito de aplicación del concepto de variable sociolingüística más allá de la fonética. Dicha extensión fue cuestionada por Lavandera (1978), arguyendo que no estaba garantizado el principio de invariabilidad semántica (Labov 1966) en el estudio de variables sintácticas. No obstante, en la actualidad, se ha defendido la necesidad de relativizar la exigencia de igualdad semántica de las variantes y reemplazarla por un criterio de equivalencia funcional (Romaine 1996, Silva-Corvalán 2001 y Serrano 2011). De este modo, según Prieto (1995-1996), "el escepticismo que rodea a las posibilidades analíticas y explicativas del concepto de variable lingüística aplicado al plano sintáctico no nos debe llevar a su proscripción irrevocable” (385). López Morales (2004 y 2008), además, ha analizado -críticamente- las objeciones a la aplicación del concepto de variable al estudio de la variación sintáctica, colocando en relieve los aspectos metodológicos y epistemológicos que constituyen, a

cláusulas de complemento directo. Específicamente, se señala que "[...] se produce con frecuencia en la lengua descuidada un uso superfluo de la preposición de ante oraciones complementarias introducidas por que: Me dijeron de que saliese; Temo de que lo hagan mal [...]" (RAE 1973: 522, nota 1). El Diccionario Panhispánico de Dudas (RAE 2005), en cambio, proporciona más información al respecto, en la entrada correspondiente, en la que define el dequeísmo como "el uso indebido de la preposición de delante de la conjunción que cuando la preposición no viene exigida por ninguna palabra del enunciado" (s. v. dequeísmo). La Nueva gramática básica de la RAE (2011: 239) propone una definición muy semejante de dicho fenómeno. 
nuestro juicio, el marco más adecuado para poner en perspectiva las posturas en este debate. ${ }^{3}$

\section{Marco conceptual}

Gómez Torrego (1999) caracteriza al dequeísmo, desde el punto de vista gramatical, en contraste con el queísmo. Según este autor, el dequeísmo consiste en "[...] el empleo de la preposición de delante de la conjunción subordinante que cuando aquella es superflua en el contexto en que aparece, es decir, cuando ningún elemento de la oración en la que se encuentra la exige" (1999: 2105). Tanto “dequeísmo" como “queísmo" deberían ser considerados como términos neutros que describen esquemas sintácticos en la subordinación, de acuerdo con Gómez Torrego (1999). ${ }^{4}$

\subsection{Los estudios sobre el desqueísmo en muestras orales}

Quien analizó la presencia del dequeísmo y el queísmo en la norma culta de Santiago de Chile, en primer lugar, fue Rabanales (1974). Según este autor, el dequeísmo consiste en la “[...] tendencia a

\footnotetext{
3 Con respecto a esto, resulta de gran interés la revisión conceptual y metodológica de Caravedo (2003), para quien la raíz fundamental del problema del concepto de variable no es la extensión de la equivalencia semántica al plano de la sintaxis, sino que la noción misma de equivalencia de significado como requisito exclusivo de las variables. En palabras de Caravedo, "Si bien no cabe duda de que el manejo de variantes con significado equivalente resulta menos complicado desde el punto de vista metodológico, no es la equivalencia el principio determinante de la variación misma. Es más, la posibilidad de surgimiento de distintos valores funcionales debe considerarse también como parte de la condición de todo hecho variable" (2003: 544). De modo consecuente, la autora distingue entre variación no funcional (sin cambio de significado) y variación funcional (con cambio de significado), con el propósito de complementar la dirección analítica del estudio de la variación lingüística, centrada en los procesos microcontextuales, con una dirección sintética, que aborde además los macrocontextos en los que se produce la variación.

4 Con respecto a las causas lingüísticas del dequeísmo, Gómez Torrego (1999) menciona la analogía o el cruce de estructuras (Rabanales 1974), la ultracorrección de grupos sociales medios por imitación de los de mayor prestigio (Bentivoglio 19801981) y el carácter vacío de la preposición $d e$, que es excesivamente polisémica, lo que la convierte en un nexo prácticamente vacío (Seco 1989 y Rabanales 1974).
} 
anteponer la preposición de al que preferentemente gramemático, cuando la norma 'oficial' no hace esperar su presencia” (1974: 415). Por otro lado, sostiene que el dequeísmo y el queísmo constituyen tendencias que revelan una inestabilidad normativa, debido al cruce de estructuras parecidas como: espero que venga mañana / tengo la esperanza de que venga mañana > *espero de que venga mañana (dequeísmo) / *tengo la esperanza que venga mañana (queísmo). ${ }^{5}$ Con posterioridad, el dequeísmo y el queísmo han sido abordados, en muestras orales de diferentes comunidades de habla española, en conjunto, por Bentivoglio (1976), Arjona (1978 y 1979), McLauchlan (1982), García (1986), Boretti de Macchia (1989), Carbonero (1992), Gómez Molina y Gómez Devís (1995), del Valle Rodás (1996-1997) y Almeida (2009). Por su parte, Bentivoglio y D’Introno (1977), Bentivoglio (1980-1981), Quilis (1986), Prieto (1995-1996) y Serrano (1998) tratan el dequeísmo, de manera independiente. ${ }^{6}$ Almeida (2009) compara los resultados de la mayoría de los estudios sobre el dequeísmo y el queísmo, a fin de valorar su extensión en Hispanoamérica, y concluye que el primero está en clara desventaja en relación con el segundo, pues en gran parte de las comunidades hispanohablantes el uso del dequeísmo es minoritario (25).

\subsection{Definición de la variable en estudio y criterios analíticos aplicados}

El dequeísmo es una variable que consiste en la aternancia de dos variantes, es decir, la ausencia (no dequeísmo) o la presencia

5 Rabanales destaca el carácter altamente polisémico de la preposición de, en particular, y de las preposiciones, en general, por lo que resulta anodina su presencia o ausencia (1974: 442). También señala la inconsistencia de ambos fenómenos (418), puesto que no solo coexisten en una misma comunidad, sino que también en un mismo individuo. En los casos en que no hay alternancia, según este autor, predomina claramente el queísmo sobre el dequeísmo, ya que este último rasgo tiene una marcada connotación de vulgaridad entre las personas cultas.

6 Un antecedente directo de nuestro estudio es el artículo de Prieto (1995-1996), en el que informa los resultados de dos indagaciones sobre el dequeísmo en el habla santiaguina: a) en una muestra de 192 entrevistas sociolingüísticas realizadas a hablantes santiaguinos y b) en una muestra de programas radiales o televisivos en los que participaron miembros de la élite política chilena. 
(dequeísmo) de la preposición de ante un que (conjuntivo o relativo) cuando no es exigida por el sistema de la lengua española, esto es, cuando es sintácticamente innecesaria, por ejemplo, le dije 0 que viniera (no dequeísmo) / ‘le dije de que viniera (dequeísmo). Una de las complejidades del estudio del queísmo y el dequeísmo radica en que en estos fenómenos se produce una intersección dinámica entre el sistema de la lengua, la norma académica y el uso concreto de los hablantes ( $c f$. Coseriu 1982: 106-107). A pesar de que en muchos estudios se invoca la norma académica como criterio para delimitar los casos de dequeísmo, en este estudio, hemos asumido una perspectiva centrada en el sistema de la lengua española como criterio, prescindiendo de un enfoque normativista. ${ }^{8}$ Desde nuestro punto de vista, en el caso del dequeísmo, lo determinante es el empleo innecesario de la preposición de ante que. Como método de comprobación para determinar la necesidad o no de la preposi-

7 Como es usual en los estudios lingüísticos, en nuestro análisis, la marca * señala que la construcción es agramatical. No obstante, nos parece oportuno insistir en que la noción de agramaticalidad que aquí manejamos se refiere a una inconsistencia, de acuerdo con el sistema de la lengua española, no como una incorrección, con respecto al dictamen de la norma académica. A este propósito, cabe precisar que por "inconsistencia" nos referimos a un juicio descriptivo que indica una incoherencia con las reglas del sistema lingüístico, mientras que con "incorrección" aludimos a un juicio prescriptivo de corte valorativo. Por otro lado, en estricto rigor, el término dequeísmo se refiere solo a la variante marcada de una variable que implica un caso de alternancia. Sin embargo, hemos optado por emplear el término dequeísmo para denominar también a la variable en estudio, ya que es muy frecuente - $y$ hasta usual - asociar la denominación de algunos fenómenos, como el que aquí nos ocupa, con su opción o variante marcada. Los alcances del concepto de variable sociolingüistica son desarrollados, en detalle, en Labov (1983, 2001 y 2004), Silva-Corvalán (2001), Caravedo (2003), López Morales (2004), Blas Arroyo (2005) y Moreno Fernández (2009).

8 Por ello, en nuestra definición del dequeísmo, hemos evitado términos como "canónico" o "no canónico". Gómez Torrego (1999) ha insistido en la necesidad de incorporar una perspectiva sistémica, en el análisis del queísmo y el dequeísmo, que supere un enfoque centrado solo en la norma académica. Asimismo, Rabanales (1974), pese al empleo del concepto de "norma oficial" en su definición de estas tendencias, en la práctica, es plenamente coherente con una aproximación al queísmo y el dequeísmo, con base en el funcionamiento del sistema lingüístico español. La noción de sistema, que aquí invocamos para el análisis del dequeísmo, remite al comportamiento de la preposición de dentro de esquemas sintácticos en el marco de la subordinación (Gómez Torrego 1999). 
ción, se empleará la sustitución de la subordinada sustantiva por un sintagma nominal y, finalmente, por un pronombre neutro. ${ }^{9}$

Otro aspecto que ha sido ampliamente debatido, en relación con el dequeísmo, es la hipótesis funcionalista, que atribuye un cambio de significado al empleo de la preposición de. En consecuencia, en el caso del dequeísmo, no podría aplicarse el concepto de "variable sociolingüística", ya que -en estricto rigor, siguiendo el principio laboviano- no constituirían dos maneras diferentes de decir lo mismo. En primer lugar, Bentivoglio y D'Introno (1977) postulan que la presencia de de ante una cláusula encabezada por que atenúa o debilita la aserción expresada en dicha cláusula. A continuación, García (1986) sostiene que sirve para que el hablante establezca una distancia - y, por lo tanto, una falta de compromiso- con respecto al contenido de la cláusula. De Mello (1995), asimismo, señala que proporciona cierta independencia a la cláusula, en relación con la oración principal, por lo que sería un recurso de enfásis. Finalmente, Schwenter (1999) y Guirado (2006) proponen que la preposición de indica que la fuente de la información no es el hablante; en consecuencia, constituye una marca de evidencialidad. ${ }^{10}$ Ante esta hipótesis surge como cuestionamiento la dificultad del investigador para determinar cuáles son, en realidad, las intenciones pragmáticas que tendría el hablante para utilizar una u otra variante. Como bien señala López Morales (2004 y 2008), las supuestas diferencias semánticas o pragmáticas entre las variantes de una misma variable deben comprobarse no atendiendo de modo exclusivo a la introspección

\footnotetext{
9 Cabe recordar que, en lo sustancial, este método es el que sugiere Rabanales (1974) y, posteriormente, Gómez Torrego (1999). También se utilizará como método la sustitución con una cláusula de infinitivo y la transformación a una oración interrogativa; este último es recomendado por el Diccionario Panhispánico de Dudas (RAE 2005). Rabanales (1992) ha sido de gran utilidad para la comprensión de un método que nos permita delimitar el concepto de dequeísmo, desde el punto de vista de la gramática científica. De igual manera, en nuestro análisis, consideraremos los casos especiales de verbos y locuciones con dos regímenes y de verbos en los que la diferencia de régimen conlleva diferencias de significado, señalados por Gómez Torrego (1999).

10 Boretti de Macchia (1989), Carbonero (1992), Del Valle Rodás (1996-1997) y Serrano (1998) también adhieren a la hipótesis funcionalista, aunque con diferentes matices.
} 
del analista, sino que recurriendo también a pruebas empíricas o, al menos, a análisis contextuales rigurosos. Debido a que la aplicación de dichas pruebas excede los objetivos que aquí nos hemos propuesto, en nuestro análisis, intentaremos determinar si la hipótesis funcionalista es plausible mediante el estudio de los contextos de ocurrencia del dequeísmo, tal como lo realiza Almeida (2009).

\section{Metodología}

\subsection{Corpus}

El corpus que servirá de base para nuestro estudio corresponde a 120 entrevistas sociolingüísticas pertenecientes a la muestra de Santiago del corpus del grupo de Estudio Sociolingüístico del Español de Chile (ESECH). ${ }^{11}$ El número de entrevistas antes indicado totaliza, aproximadamente, 116 horas de grabación, cuya transcripción fue revisada por completo.

\subsection{Población y muestra}

En nuestra investigación se consideró la población constituida por hombres y mujeres de la Región Metropolitana de más de 20 años de edad. ${ }^{12}$ El cuestionario se aplicó a una muestra del tipo "muestra por cuotas

11 El ESECH es un grupo de investigación del Departamento de Lingüística de la Facultad de Filosofía y Humanidades de la Universidad de Chile coordinado por nosotros. Las entrevistas fueron realizadas a hablantes santiaguinos, entre 2005 y 2011, por los estudiantes en la asignatura de Sociolingüística de los programas de Licenciatura en Lengua y Literatura Hispánicas y Licenciatura en Lengua y Literatura Inglesas de la Universidad de Chile. En la situación de entrevista, los entrevistadores debían tratar de superar la "paradoja del observador" consiguiendo, de esta forma, una muestra significativa de discurso natural grabado (estilo vernáculo) de hablantes representativos de la comunidad de habla en estudio (Labov 1983).

${ }_{12}$ Por lo que refiere a los criterios de asignación de estatus de hablante nativo de Santiago de Chile, hemos adaptado la propuesta de Prieto (1995-1996), por lo que se aplicaron las siguientes restricciones en la selección de los sujetos: 1) haber nacido y residido en forma ininterrumpida en Santiago, 2) haber nacido y residido en Santiago la mayor parte de sus vidas, salvo por periodos que sumados no superen los cinco años y 3) haber residido en forma ininterrumpida en Santiago desde los cinco años de edad. 
con afijación uniforme” (López Morales 1994: 58). La muestra, así conformada, comprende un total de 120 entrevistas realizadas a igual número de sujetos, distribuidos como se indica en la tabla 1 que mostramos a continuación: ${ }^{13}$

Tabla 1. Proporción de sujetos según características sociodemográficas de sexo, edad y grupo socioeconómico

\begin{tabular}{lccccccc}
\hline & \multicolumn{2}{c}{$20-34$} & \multicolumn{2}{c}{$35-54$} & \multicolumn{2}{c}{55 y más } & \multirow{2}{*}{ Total } \\
\cline { 2 - 6 } & $\mathrm{H}$ & $\mathrm{M}$ & $\mathrm{H}$ & $\mathrm{M}$ & $\mathrm{H}$ & $\mathrm{M}$ & \\
\hline Medio alto & 5 & 5 & 5 & 5 & 5 & 5 & $=30$ \\
Medio & 5 & 5 & 5 & 5 & 5 & 5 & $=30$ \\
Medio bajo & 5 & 5 & 5 & 5 & 5 & 5 & $=30$ \\
Bajo & 5 & 5 & 5 & 5 & 5 & 5 & $=30$ \\
& 20 & 20 & 20 & 20 & 20 & 20 & $=120$ \\
\hline
\end{tabular}

3.3. Procedimiento de estratificación social utilizado en ESECH

Para la estratificación de los sujetos que conforman la muestra del estudio, se empleó el sistema de adscripción de estatus social empleado en ESECH que se basa en la asignación del puntaje -que se indica entre paréntesis - a los informantes, de acuerdo con las siguientes variables: a) nivel educacional (3), b) profesión u ocupación (2) y c) comuna de residencia (1). ${ }^{14}$ A partir de la asignación de los mencionados puntajes, se distinguieron cuatro

13 En cuanto al tamaño de la muestra, hemos optado por conformarla con un número de informantes considerado, en general, como suficiente en la bibliografía sociolingüística (cf. Trudgill y Hernández Campoy 2007: s. v. representatividad y López Morales 1994: 52).

${ }_{14}$ La explicación en detalle de dichas variables se encuentra en Prieto (1995-1996), cuya propuesta fue perfeccionada y actualizada con base en los estudios disponibles acerca de la realidad socioeconómica chilena (San Martín y Guerrero 2015). Cabe precisar que el modelo de nivel socioeconómico fue adaptado de estos estudios de mercado en función de los objetivos que se propone el grupo ESECH, que busca definir un perfil más bien sociocultural de la comunidad lingüística en estudio. En consideración con esto, para la estratificación socioeconómica, una de las variables más determinantes fue el nivel educacional y, por esta razón, es la que asigna el mayor puntaje. Asimismo, el nivel de ingresos demuestra muy poca relevancia para los estudios antes mencionados, por ello no fue considerado en la determinación de los perfiles socioeconómicos. 
grupos socioeconómicos, definidos según los rangos siguientes: a) Medio alto (MA): 42 - 36, b) Medio (M): 35-27, c) Medio bajo (MB): $26-18$ y d) Bajo (B): 17 - 6. El intervalo de cada rango responde a la mayor coincidencia con los puntajes que han establecido los estudios sociológicos y de mercado para la clasificación de estratos socioeconómicos, aunque con las debidas adaptaciones, puesto que en esta muestra no se incluyen ni el grupo de la extrema pobreza ni el de la extrema riqueza ( $c f$. San Martín y Guerrero 2015).

\subsection{Procedimiento analítico}

Nuestro análisis del dequeísmo se concentró tanto en la determinación de los contextos sintácticos en los que se emplea dicha variante con mayor frecuencia, como en la observación de la incidencia de los factores sociodemográficos sexo, edad y grupo socioeconómico de los hablantes en el uso de las variantes no dequeísta y dequeísta de la variable analizada. El análisis estadístico atenderá a dos niveles de observación: a) en términos descriptivos, según frecuencias absolutas y porcentajes de frecuencia y $b$ ) en términos interpretativos o inferenciales, con base en la comparación entre medias o tendencias centrales. ${ }^{15}$ El paquete estadístico al que hemos recurrido para la estadística inferencial es el SPSS (Statistical Package for the Social Sciences), versión 15.0 para Windows, específicamente, la prueba Análisis de varianza ANOVA. En atención a que la distribución de los datos, en algunos casos, pudiera ser anormal, se complementará dicho análisis con su análogo de tipo no paramétrico, esto es, Anova de Kruskal Wallis. ${ }^{16}$

\section{Presentación y análisis de los resultados}

En nuestro corpus, se identificaron 7747 casos de alternancia de la variable "ausencia o presencia de la preposición de ante un que

15 Por lo que se refiere a la descripción de los procedimientos estadísticos aplicados, en este trabajo seguimos las sugerencias e indicaciones de Moreno Fernández (1990), López Morales (1994) y Hernández Campoy y Almeida (2005).

16 En ambos casos, el grado de significación se definirá en el $5 \%$, según el cual $\mathrm{p}=<0,05$ será estadísticamente significativo. 
conjuntivo o relativo cuando es gramaticalmente innecesaria”. De dicho total, $7576 / 7747(97,8 \%)$ casos corresponden a la variante no dequeísta, es decir, a la ausencia de la preposición de, mientras que $171 / 7747(2,2 \%)$ ocurrencias corresponden a la variante dequeísta, esto es, a su presencia. Por lo tanto, en la muestra analizada, la frecuencia del dequeísmo es abrumadoramente inferior en comparación con la del no dequeísmo, tal como puede apreciarse en el gráfico 1:

\section{Gráfico 1. Porcentaje de frecuencia de las variantes no dequeísta y dequeísta en el corpus}

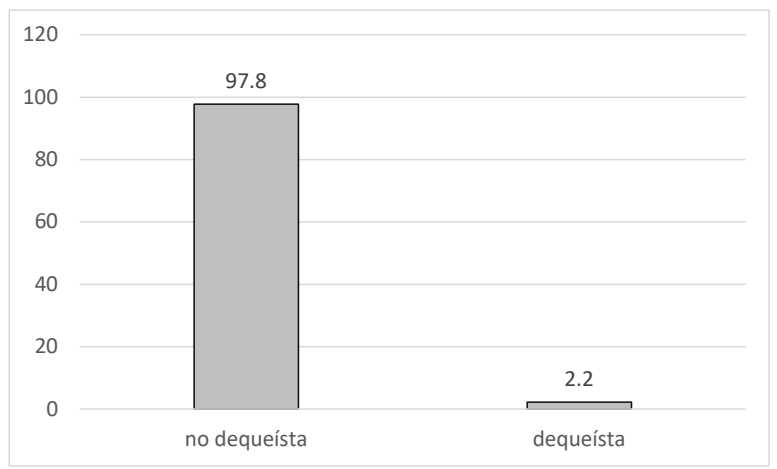

Los siguientes ejemplos ilustran la ocurrencia de la variante no dequeísta en nuestros materiales:

(1) y resulta que en aquella época los colegios particulares/ eran examinados por por los liceos (MAIIIH179), ${ }^{17}$

17 Respecto de la transcripción de los ejemplos, es necesario señalar las siguientes convenciones gráficas: 1) como se trata de variables no fonéticas y, por lo tanto, la pronunciación no es el foco de nuestro análisis, el texto correspondiente a cada ejemplo se transcribe en ortografía convencional, incluidos los acentos gráficos; 2) con el propósito de no desnaturalizar demasiado la representación del habla chilena, se ha conservado la manifestación gráfica de determinados usos característicos del español hablado en Chile; 3) para la mejor comprensión de los ejemplos incluidos en este estudio, se ha suprimido el sistema de etiquetas, que se emplea en la transcripción de las entrevistas de ESECH, excepto las pausas que se señalan con /. Al final de cada ejemplo, se indica, entre paréntesis, el código del informante, según 
(2) yo diría que mayoritariamente sí ee/ ee bueno obviamente han han ha habido ee cambios arquitectónicos (MAIIIH177),

(3) un tío recuerdo que/ contaba una historia/ que fue/ fue a cazar ee y dice que/ él vio un platillo/ con sus amigos que estaban cazando en la noche (MIIH113),

(4) lo que pasa es que la idea yo creo es pasarlo bien y no tener responsabilidad (MIIH115),

(5) sí/ siempre los trato de tú/ a no ser que no tenga mucha confianza con ellos (MIM107),

(6) la gente que ya lleva un tiempo trabajando tiene sus códigos y tiene sus formas (MAIIIH177).

Por su parte, los siguientes fragmentos ejemplifican el empleo de la variante dequeísta, en el corpus:

(7) bueno/ al principio/ se echaron de menos las amarillas porque/ resulta de que uno estaba acostumbrado a ellas (MBIIIH083),

(8) la muestra que había sido ee ee analizada digamos por un/ por un experto en estos temas y lo llevamos a otros/ esos otros dijeron de que/ ee no teni/ no estaban seguros digamos de que sí tenía cáncer/ que estaba muy en el límite (MAIIIH179),

(9) usted ve que aquí mismo ahora este dieciocho me tocó estar acá ¿cierto? /entonces/ qué pasaba/ de que el dieciocho lo hac/ lo trabajé todo el día aquí po sí me fui en la tarde (MBIIIM093),

(10) me gustan los edificios// pero en realidad Ñuñoa/ se caracteriza por ser una comuna así/ de que o sea/ no sé// es como una comuna de casas/ de abuelitos (MAIM155),

las siguientes convenciones: grupo socioeconómico ( $\mathrm{MA}=$ medio alto, $\mathrm{M}=$ medio, $\mathrm{MB}=$ medio bajo, $\mathrm{B}=$ bajo), grupo etario (III = adultos mayores de 55 años y más, $\mathrm{II}=$ sujetos adultos de edad intermedia entre 35 y 54 años y $\mathrm{I}=$ hablantes jóvenes de entre 20 y 34 años) y sexo $(\mathrm{M}=$ mujer y $\mathrm{H}=$ hombre $)$. A continuación del sexo se señala el número correlativo del sujeto en el corpus. 
(11) también es un diario de corte liberal también pro empresarial y el resto de la prensa en un/ de que no existe (MAIH145).

En nuestros materiales, no se detectaron diferencias de significado entre las variantes, no dequeísta y dequeísta, de la variable. A nuestro juicio, no existen razones de peso que nos permitan comprobar una hipótesis de tipo funcionalista acerca de las diferencias semánticas o pragmáticas entre ambas. El hecho de que - en gran medida - la ocurrencia de la variable se produzca en contextos sintácticos con verbos conjugados en tiempo presente y en primera persona del singular guarda relación con las caraterísticas de la entrevista como instrumento. No obstante, también es posible hallar ejemplos de ocurrencia de ambas variantes en estructuras sintácticas con verbos conjugados en otros tiempos verbales y con otras personas gramaticales. Por otro lado, los ejemplos anteriores reflejan cierta inestabilidad en el empleo de la preposición de delante de un que conjuntivo o relativo en algunos sujetos de la muestra. La gran mayoría de los casos de dequeísmo en el corpus corresponde al empleo de de delante de un que conjuntivo. La proporción de los casos de dequeísmo con que relativo es bastante menor. Los ejemplos 12-13 ilustran la inconsistencia en el uso de la preposición de delante de que en un mismo sujeto:

(12) yo creo que hoy día tenemos tenemos más humedad y por lo tanto ha ido cambiando el clima/ también creo de que/ en ciertos momentos ha habido más lluvias que en otro digamos/ pero eso también es una cosa cíclica de la naturaleza (MAIIIH179),

(13) y resulta de que/ yo con la impotencia que porque ella no la abrazaba porque no la tenía en sus brazos y resulta que voy llegando a un metro de donde está ella y una vecina me dice que no que no era mi hija (BIIM025). 
Asimismo, 14 constituye un caso de “deísmo", es decir, de ocurrencia redundante o gramaticalmente innecesaria de la preposición de en un contexto en el que no existe un que conjuntivo, que también nos parece que es un indicio de la inestabilidad en el uso preposicional en algunos de los sujetos:

(14) tengo que ser demasiado responsable para el trabajo entonces si yo sé que tengo que trabajar/ no/ prefiero de quedarme en la casa/ porque yo digo para ir un rato// después al otro día amanecer (MBIIIM093).

Gómez Torrego (1999) y Almeida (2009) destacan la escasa difusión del dequeísmo en la actualidad, en comparación con el queísmo. A pesar de su escasa frecuencia, el dequeísmo constituye una variable sintáctica cuyo comportamiento resulta interesante, debido a que, por un lado, se trata de un fenómeno que ha sido estudiado latamente en la lingüística hispánica y, por otro, porque se lo ha tipificado como un posible cambio lingüístico en marcha. En relación con los datos de Prieto (1995-1996), los nuestros evidencian un ligero avance del dequeísmo desde los noventa, lo que nos lleva a suponer que se trata de un rasgo más bien estable.

\subsection{Factores lingüísticos}

En este apartado, nos referiremos a los factores lingüísticos que propician el empleo del dequeísmo en la muestra analizada en esta indagación. En nuestros materiales se verificó la ocurrencia del dequeísmo en las siguientes estructuras sintácticas: 1) en una cláusula con la función de sujeto, 2) en una cláusula con la función de objeto directo, 3) en una cláusula con la función de atributo, 4) en estructuras apositivas, 5) en locuciones conjuntivas o adverbiales, 6) como sustitución de de por otras preposiciones regidas y 7) como adición de de con que relativo. En la tabla 2 y el gráfico 2 se muestran las frecuencias absoluta y porcentual de la ocurrencia del dequeísmo en nuestro corpus: 
Tabla 2. Frecuencia absoluta y porcentaje de las variantes no dequeísta y dequeísta en los contextos identificados en la muestra

\begin{tabular}{lccc}
\hline Contexto & No dequeísmo & Dequeísmo & Totales \\
\hline 1) sujeto & $1814(98,2)$ & $32(1,8)$ & $1846(100)$ \\
2) objeto & $4419(98)$ & $89(2)$ & $4508(100)$ \\
3) atributo & $469(99,8)$ & $1(0,2)$ & $470(100)$ \\
4) apositiva & $98(92,5)$ & $8(7,5)$ & $106(100)$ \\
5) locuciones & $223(95,3)$ & $11(4,7)$ & $234(100)$ \\
6) otras preposiciones & $173(92,5)$ & $14(7,5)$ & $187(100)$ \\
7) que relativo & $380(96)$ & $16(4)$ & $396(100)$ \\
\hline
\end{tabular}

Gráfico 2. Porcentaje de frecuencia de las variantes no dequeísta y dequeísta en los contextos identificados en la muestra

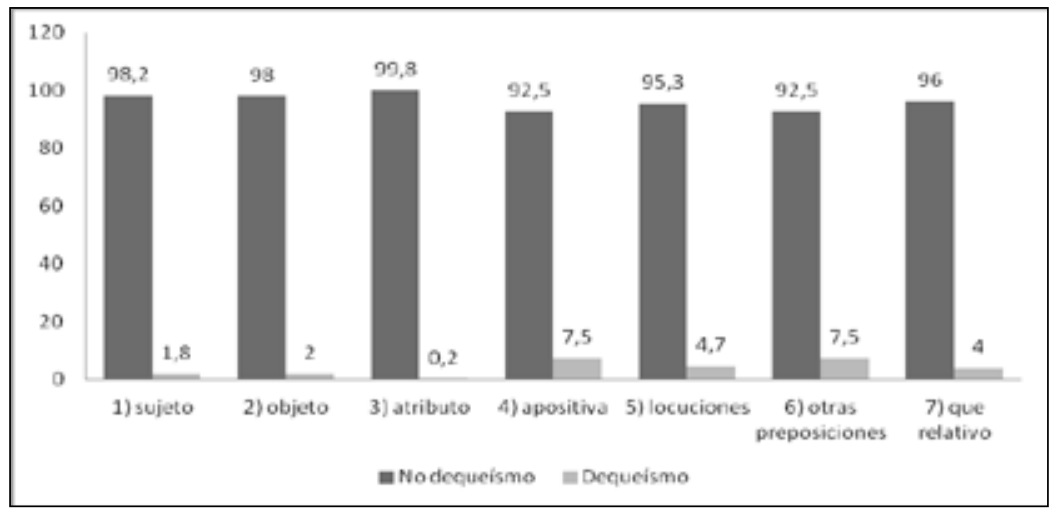

En términos relativos, los contextos lingüísticos que más favorecen la ocurrencia del dequeísmo son la sustitución de otras preposiciones regidas por de y la adición de de en estructuras apositivas. Le siguen en importancia el empleo del dequeísmo en locuciones conjuntivas y adverbiales y con que relativo. Por su parte, la presencia de la preposición de delante de un que conjuntivo en cláusulas con la función de objeto directo y de sujeto muestran una frecuencia relativa menor. Finalmente, el dequeísmo en cláusulas de atributo prácticamente no fue relevado en nuestros 
materiales. A pesar de estos porcentajes, es importante destacar que, en términos de frecuencia absoluta, la mayoría de los casos de dequeísmo del corpus se encontraron en cláusulas con la función de objeto directo y, de modo secundario, con la función de sujeto.

A continuación, se ejemplificará y explicará cada contexto en que se verificó la incidencia de la variante dequeísta:

1) En una cláusula con la función de sujeto

Se trata del tipo de dequeísmo que ocupa el segundo lugar en importancia con respecto a su frecuencia absoluta. En este contexto sintáctico destacan el empleo del verbo resultar + de que (15-16) y el dequeísmo en las cláusulas sujeto en oraciones copulativas (1718). Finalmente, 19 es un caso de uso expletivo de la preposición $d e$ con un verbo doblemente pronominal: "olvidársele a alguien que". Ejemplos:

(15) y ahí completé/ porque resultó de que yo hice los primeros/ ee cursos/ en y después ingresé al a la mm ¿cómo se llama?/ ésta la Escuela ES (MBIIIH083),

(16) y resulta de que no pude ir porque andaba ap/ aparte de eso que andaba con pantalones claros se me notaba mucho/ así que tuve que tomar un taxi/ y irme a la casa de mi abuela (BIIM028),

(17) podría ser de que al tener neumonía/ la doctora a uno le plantea todos los los posibles estados que pueden ser (MAIIIH180),

(18) es innegable/ de que el calentamiento global también nos afecta// y tal vez donde más se ve es en el derretimiento de los hielos/ polares (MAIIH164),

(19) pero después en el trascurso del día con la moviendo moviendo el organismo/ después hasta/ se hasta se le olvida a uno de que de que tiene algún problemita po (MBIIIH083). 
2) En una cláusula con la función de objeto directo

Según la tabla 2, este tipo de construcción dequeísta resultó ser la más frecuente en términos absolutos. En esta estructura destaca el empleo de verbos de comunicación (20-22), en especial, decir y de verbos de pensamiento (23-24), si bien también se halló dequeísmo con verbos de sentimiento (25), voluntad (26) y percepción (27). Ejemplos:

(20) mira/ yo en realidad ee yo podría decir de que mi profesión es/ profesor universitario/ pero de/ bajo el punto de vista de/ una carrera universitaria/ yo soy constructor civil (MAIIIH179),

(21) hay una deformación del lenguaje y ee/ lamentablemente/ por más que el/ se diga de que la gente o el rotito chileno habla ee/ de una manera en particular/ (MIIIH130),

(22) como que se puso a alegar de que que que por culpa de que el portón está malo qué sé yo y todo entraron (MAIIIM186),

(23) la cuestión/ es que yo recuerdo/ de que mi abuelito/ que había fallecido ee a ver/ el año cincuenta y ocho falleció él (MIIIH130),

(24) porque encuentro de que son muy caras las entradas y otra de que no hay seguridad en los estadios (BIIIH033),

(25) me molesta también el hecho de que una pareja sea idiota/ me molesta mucho/ de que sea idiota sea estúpida o estúpido (MIH097),

(26) y ellos nos pedían de que les indicáramos donde estaba/ la sala de recepción que era justamente una sala especial (MBIIIH083),

(27) he escuchado de que ee ee es bastante común ee aún la cantidad de asaltos que se producen en el centro de Santiago (MAIIIH177). 
3) En una cláusula con la función de atributo

Solo se encontró un caso de dequeísmo que podría adscribirse a esta categoría. No obstante, se trata de un caso dudoso, ya que también podría corresponder a una estructura de cláusula de sujeto. De todas formas, lo contabilizamos como tal, porque otros estudiosos del dequeísmo lo considerarían una estructura atributiva. Ejemplo:

(28) lo que pasa es de que ee desde que hace muchos años que el mes de María se hace acá (MIIIM137).

4) En estructuras apositivas

La ocurrencia de este tipo de dequeísmo fue escasa en nuestro corpus, ya que se identificaron solo ocho casos, si bien se trata de uno de los contextos que más predispone la ocurrencia del fenómeno en términos relativos. Ejemplo:

(29) esa era la idea en ese tiempo/ de que en el colegio nos conseguían un lugar donde hacer la práctica y uno luchaba por quedar contratada (MBIIM074).

(30) viernes en la noche o sábado o domingo/ de que ya no ya no iba al colegio uno po/ entonces qué pasaba/ de que usted salía a la puerta afuera/ uno jugaba a las naciones/ jugaba al pillarse/ y jugaba al corre el anillo (MBIIIM093).

5) En locuciones conjuntivas o adverbiales

Se trata, en lo principal, de usos expletivos de la preposición de delante de que en las conjunciones de tal manera que (31) y a no ser que (32). 33, para finalizar, corresponde a un dequeísmo con un adverbio en -mente. Ejemplos:

(31) el lugar también está muy bien organizado/ de tal manera de que segmen/ han segmentado e o/ niños/ lolos adolescentes/ o sea/ no a adolescentes y adultos/ digamos (MAIIIH180), 
(32) puesto que son ellos los únicos que nos pueden/ trasladar/ a no ser de que uno tuviese su locomoción propia (MIIIH132),

(33) ee sí/ bueno por temas de trabajo cuando se tiene// bueno ee ee cosas no no demasiado distintas a la a a la vida normal/ solamente de que ee uno sabe que tiene ee todo el tiempo disponible para hacer lo que uno desea hacer (MAIIIH177).

6) Como sustitución de de por otras preposiciones regidas

En general, se identificaron sustituciones de las preposiciones en (34) y por (35) por de. También es una estructura que favorece el empleo del dequeísmo de modo relativo. Ejemplos:

(34) por supuesto que sí/ eso ha influido de que no tengamos que ir a a lo cercano de la comuna/ al centro de la comuna y tampoco al centro de Santiago porque ee ya no necesitas ir tan lejos a comprar tus cosas/ las tienes a la mano (MIIIH131),

(35) como a todos los pobres po/ o sea tener que esperar estar en el suelo muriéndome y salió una enfermera a retarme de que estaba haciendo show (MBIH052).

7) Como adición de de con que relativo

En este contexto el carácter expletivo de la preposición de es bastante evidente, como puede apreciarse en 36-37. Ejemplos:

(36) en general amigos del trabajo/ del trabajo/ o de que por alguna circunstancia tú te los has ido tomando en la vida pero que en general tienen que ver con tu vida laboral o derivan de eso (MAIIM173),

(37) claro es ahí está la diferencia/ que tengo amigos de que los veo todos los días que son de la parte laboral (MBIIH065). 
Aparte de los contextos lingüísticos previamente expuestos, nuestro análisis de los factores internos del dequeísmo incluyó el inventario de los verbos que promovían su ocurrencia. En la tabla 3, se muestran las frecuencias absoluta y porcentual de 47 verbos con los cuales se verificó la presencia expletiva de la preposición de ante la conjunción que:

Tabla 3. Frecuencia absoluta y porcentaje de los verbos dequeístas identificados en la muestra

\begin{tabular}{llc}
\hline Verbos & Frecuencia absoluta & Porcentaje de frecuencia \\
\hline decir & 19 & 15 \\
\hline ser & 16 & 12,6 \\
resultar & 12 & 9,5 \\
\hline creer & 9 & 7,1 \\
\hline pensar & 9 & 7,1 \\
ver & 5 & 3,9 \\
pasar & 4 & 3,1 \\
\hline saber & 4 & 3,1 \\
encontrar & 3 & 2,4 \\
esperar & 3 & 2,4 \\
\hline considerar & 2 & 1,5 \\
\hline escuchar & 2 & 1,5 \\
hacer & 2 & 1,5 \\
molestar & 2 & 1,5 \\
olvidar & 2 & 1,5 \\
recordar & 2 & 1,5 \\
aclarar & 1 & 0,8 \\
alegar & 1 & 0,8 \\
avisar & 1 & 0,8 \\
cachar & 1 & 0,8 \\
captar & 1 & 0,8 \\
\hline coincidir & 1 & 0,8 \\
comentar & 1 & 0,8 \\
\hline comprender & 1 & 0,8 \\
\hline
\end{tabular}




\begin{tabular}{lcc}
\hline Verbos & Frecuencia absoluta & Porcentaje de frecuencia \\
\hline comprobar & 1 & 0,8 \\
contar & 1 & 0,8 \\
criticar & 1 & 0,8 \\
dar & 1 & 0,8 \\
\hline darse & 1 & 0,8 \\
\hline deducir & 1 & 0,8 \\
dejar & 1 & 0,8 \\
\hline desmentir & 1 & 0,8 \\
enseñar & 1 & 0,8 \\
entender & 1 & 0,8 \\
hablar & 1 & 0,8 \\
implicar & 1 & 0,8 \\
mentir & 1 & 0,8 \\
necesitar & 1 & 0,8 \\
opinar & 1 & 0,8 \\
pedir & 1 & 0,8 \\
permitir & 1 & 0,8 \\
predicar & 1 & 0,8 \\
reclamar & 1 & 0,8 \\
sentir & 1 & 0,8 \\
temer & 1 & 0,8 \\
tocar & 1 & 0,8 \\
vivir & 1 & 0,8 \\
Total & 127 & 100 \\
\hline & & \\
\hline & & \\
\hline
\end{tabular}

Los verbos que concentran mayormente los casos de dequeísmo son decir, ser y resultar, seguidos a gran distancia por creer y pensar, así como - con una menor frecuencia- por ver, pasar, saber, encontrar, esperar, considerar, escuchar, hacer, molestar, olvidar y recordar. Otros verbos aportan un único caso de adición redundante de la preposición de delante de que. Estos verbos se clasificaron en siete categorías, las que se presentan, a continuación, en orden de frecuencia, respecto del total (127): 
a) Cognición: se refieren a procesos mentales o de entendimiento. Captar, cachar, creer, comprender, comprobar, considerar, coincidir, deducir, encontrar, enseñar, entender, implicar, olvidar, pensar, recordar y saber (16 unidades que suman 40 casos con el 31,5\%).

b) Enunciación: aluden a actos verbales o de comunicación. Aclarar, alegar, avisar, comentar, contar, criticar, decir, desmentir, hablar, mentir, opinar, predicar y reclamar (13 unidades que llegan a 31 ocurrencias y al 24,4\%).

c) Voluntad: destacan el carácter volitivo o intencional de las acciones. Dar, dejar, esperar, hacer, necesitar, pedir y permitir (siete unidades que suman 19 casos con el 15\%).

d) Cópula verbal: verbo copulativo. Ser (un verbo con 16 casos y el $12,6 \%)$.

e) Acontecimiento: colocan de relieve la ocurrencia de hechos o eventos. Darse, pasar, resultar, tocar y vivir (cinco unidades que concentran 10 casos con el 7,9\%).

f) Percepción: aluden a acciones de percepción sensible. Escuchar y ver (dos unidades que aportan siete ocurrencias y el 5,5\%).

g) Emoción: expresan sentimientos o afectos. Molestar, sentir y temer (tres verbos que suman cuatro casos con el 3,1\%).

También se analizó la forma personal o no personal de los verbos con los cuales ocurrió el dequeísmo en nuestros materiales. A este respecto, los resultados indican que en 80/127 ocasiones los verbos en construcciones dequeístas aparecen conjugados en forma personal $(63 \%)$. Ejemplos:

(38) yo creo de que es una herramienta que sirve/ porque la historia y más que la historia/ la memoria/ que es más algo ee como mucho más allá de la construcción histórica/ es algo peligroso (MAIH145),

(39) yo te digo que en ese sentido creo de que hay más becas yo yo/ Australia nos ofreció (MAIIIH179). 
Por el contrario, en 47/127 casos, los verbos que aparecen en estructuras dequeístas son empleados en alguna de sus formas no personales (37\%), lo que parece indicar que esta última forma no constituye un factor interno muy determinante para la ocurrencia del dequeísmo. Este resultado se reparte en las distintas modalidades de formas no personales del verbo de la siguiente manera: a) infinitivo, en $37 / 47$ ocurrencias con el $78,7 \%$; b) gerundio, en $2 / 47$ casos lo que representa tan solo el $4,3 \%$ y c) participio, en $8 / 47$ ocasiones con el $17 \%$. Ejemplos:

(40) no van a permitir de que los poderes laicos un poco más se apoderen de la educación que ellos siempre han tenido como su trinchera (MAIH145),

(41) por ahí entonces se ha producido/ ee de que no han entregado todo lo que tenían que entregar/ para que el sistema diseñado como está ¿̇no cierto?/ ee funcione (MIIIH130),

(42) lo material no/ nunca tanto apego a lo material// siempre pensando de que no los pasó nada a la familia/ en eso no más pero lo material se recupera y punto (BIIIH033).

Finalmente, se analizó la incidencia de la intercalación de elementos parentéticos, procedimiento que tampoco resultó particularmente determinante, ya que se verificó solo en 14/171 ocasiones, lo que corresponde al 8,2\%. Ejemplos:

(43) lo otro que me molesta mucho y que lo he estado comentando estos días/ayer parece que fue no más/ de que el hecho que como están muchos muchas/ mucha / mucha gente/ mucho joven ee de tipo/ ee como te dijera de/ caribeño (MAIIIH181),

(44) entonces/ eso me quedó muy grabado/ eso quiere decir/ ¿no cierto?/ de que/ es muy probable de que el número de la disposición en el caso nuestro no sea tan importante si tú sabes el concepto (MAIIIM188). 
Gómez Molina y Gómez Devís (1995) destacan la ocurrencia del dequeísmo como sustitución de de por otras preposiciones regidas y en cláusulas de sujeto y objeto directo. Por su parte, Almeida (2009) lo hace con respecto a las estructuras apositivas y las cláusulas de sujeto. De igual modo, Arjona (1979) y Del Valle Rodás (1996-1997) indican que el dequeísmo es favorecido por las cláusulas de sujeto y de objeto directo, respectivamente. Serrano (1998), asimismo, destaca el empleo del dequeísmo con verbos de comunicación y de pensamiento, por lo que propone que consiste en un marcador deíctico de expresión de opinión. Por último, en su indagación en una muestra de miembros de la élite política chilena, Prieto (1995-1996) concluye que el empleo de las formas no personales del verbo y la intercalación de elementos parentéticos son determinantes en la ocurrencia del dequeísmo. Si bien nuestros datos no respaldan dicha determinación, es importante recordar que se trata de muestras de naturaleza diferente, ya que los materiales del estudio de Prieto provenían de declaraciones radiales o televisivas de políticos de la época.

\subsection{Factores sociodemográficos}

En esta sección, se exponen los resultados de nuestro estudio con respecto a la relación de la frecuencia de empleo de las variantes no dequeísta y dequeísta con las tres variables extralingüísticas consideradas en el análisis: sexo, edad y grupo socioeconómico de los sujetos de la muestra. ${ }^{18}$

\subsubsection{Sexo}

En la tabla 4 y el gráfico 3, presentamos las frecuencias absoluta y porcentual de la ocurrencia de no dequeísmo y dequeísmo identificadas en el corpus, de acuerdo con el sexo de los sujetos.

\footnotetext{
18 Específicamente, nos interesa determinar cómo se distribuye el empleo de las variantes analizadas en la muestra, con relación a los distintos factores extralingüísticos incluidos en el estudio, a efecto de identificar cuál es el factor más influyente.
} 
Tabla 4. Frecuencia absoluta y porcentaje de las variantes no dequeísta y dequeísta en el corpus según el sexo de los sujetos de la muestra

\begin{tabular}{lccc}
\hline Sexo & No dequeísmo & Dequeísmo & Totales \\
\hline Hombres & $3624(96,9)$ & $117(3,1)$ & $3741(100)$ \\
Mujeres & $3952(98,7)$ & $54(1,3)$ & $4006(100)$ \\
\hline
\end{tabular}

Gráfico 3. Porcentaje de frecuencia de las variantes no dequeísta y dequeísta en el corpus según el sexo de los sujetos de la muestra

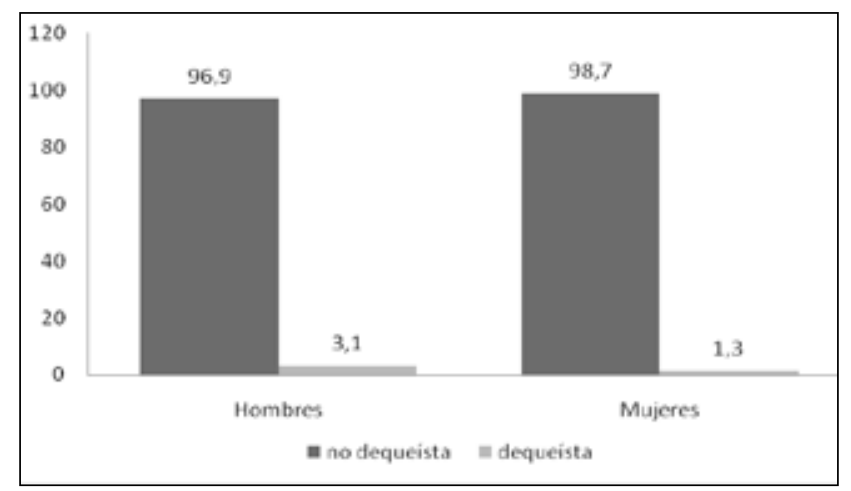

Según los datos precedentes, en términos estadísticos descriptivos, la ocurrencia de la variante no dequeísta es predominante en las mujeres que conforman la muestra bajo estudio, mientras que la de la variante dequeísta lo es en los hombres. De acuerdo con los resultados del análisis paramétrico ANOVA, la media del no dequeísmo de los hombres es 60,400 y la de las mujeres, 65,687. No obstante, los resultados no son significativos estadísticamente $(\mathrm{F}=0,93, \mathrm{p}=0,335)$. La aplicación de la prueba no paramétrica no fue necesaria en el caso del no dequeísmo, debido a que los datos se distribuyen normalmente. Por otra parte, el gráfico 4 presenta los resultados de la comparación de las medias del dequeísmo, según el sexo de los sujetos: hombres $(1,950)$ y mujeres $(0,900)$. Por lo tanto, aunque se trata de medias muy similares, se muestra una mayor tendencia hacia el dequeísmo 
por parte de los hombres. Las diferencias entre las medias reportadas son estadísticamente significativas $(\mathrm{F}=4,601, \mathrm{p}=0,034)$.

Gráfico 4. Medias marginales estimadas de dequeísta

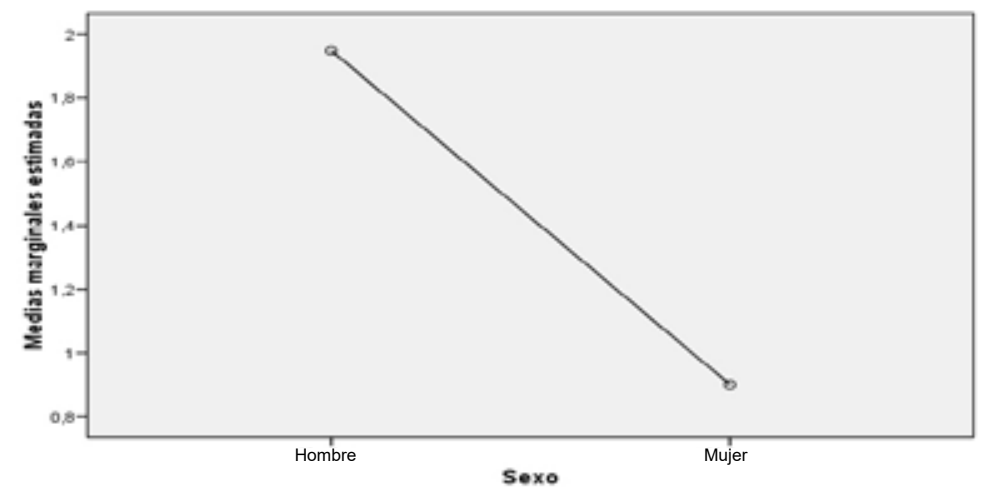

La aplicación de la prueba no paramétrica (Anova de KruskalWallis), por su lado, arroja los rangos promedio: hombres $(67,62)$ y mujeres $(53,38)$ y confirma la significatividad estadística de estas diferencias, ya que Chi-cuadrado $=5,943, p=0,015$. Por consiguiente, en términos estadísticos inferenciales, podemos confirmar el predominio del empleo del dequeísmo en los sujetos de sexo masculino.

\subsubsection{Edad}

La tabla 5 y el gráfico 5 muestran las frecuencias absoluta y porcentual de la ocurrencia de las variantes no dequeísta y dequeísta identificadas en el corpus, según la edad de los sujetos.

Tabla 5. Frecuencia absoluta y porcentaje de las variantes no dequeísta y dequeísta en el corpus según la edad de los sujetos de la muestra

\begin{tabular}{lccc}
\hline Edad & No dequeísmo & Dequeísmo & Totales \\
\hline $20-34$ & $2657(98,7)$ & $35(1,3)$ & $2692(100)$ \\
\hline $35-54$ & $2532(98,4)$ & $41(1,6)$ & $2573(100)$ \\
\hline 55 y más & $2387(96,2)$ & $95(3,8)$ & $2482(100)$ \\
\hline
\end{tabular}


Gráfico 5. Porcentaje de frecuencia de las variantes no dequeísta y dequeísta en el corpus según la edad de los sujetos de la muestra

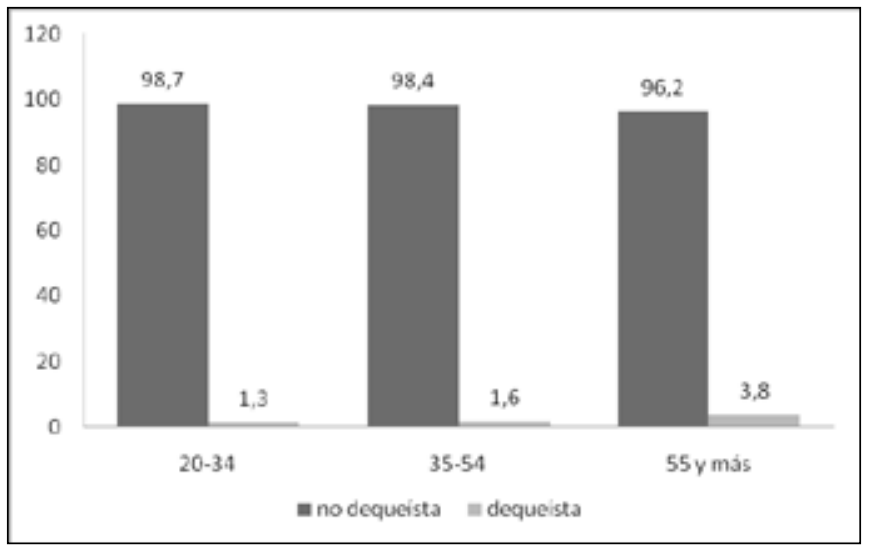

Descriptivamente, no existen diferencias en el empleo de las variantes analizadas, según los diferentes grupos etarios, si bien la variante dequeísta registra una mayor frecuencia de empleo por parte del grupo etario más viejo. Los resultados del análisis paramétrico de la ocurrencia de la variante no queísta son los siguientes: 20-34 años $(66,425), 35-54(63,300)$ y 55 y más $(59,675)$. Sin embargo, los resultados no son estadísticamente significativos $(\mathrm{F}=0,473$, $\mathrm{p}=0,624)$. Asimismo, el gráfico 6 presenta la comparación de las medias correspondientes a la variante dequeísta: $20-34$ años $(0,875)$, $35-54(1,025)$ y 55 y más $(2,375)$. Según la prueba paramétrica, se trata de diferencias estadísticamente significativas, ya que $\mathrm{F}=3,864$, $\mathrm{p}=0,024$, aunque debido a la distribución relativamente anormal de estos datos es importante confirmar el grado de significación con una prueba no paramétrica.

La prueba Anova de Kruskal-Wallis, por su parte, da los siguientes rangos promedio para las edades de los sujetos: 20-34 años $(50,85), 35-54$ (63,70), 55 y más $(66,95)$. No obstante, la misma prueba indica, aunque por poco, que estas diferencias no son significativas, puesto que Chi-cuadrado $=5,670, p=0,059$. Por lo tanto, concluimos que las diferencias de las frecuencias de la variante 
dequeísta, según la edad de los sujetos, no son significativas, aunque marcan una clara tendencia hacia el predominio del dequeísmo en los sujetos de edad avanzada, la que podría confirmarse de ampliar el tamaño de la muestra bajo análisis.

\section{Gráfico 6. Medias marginales estimadas de dequeísta}

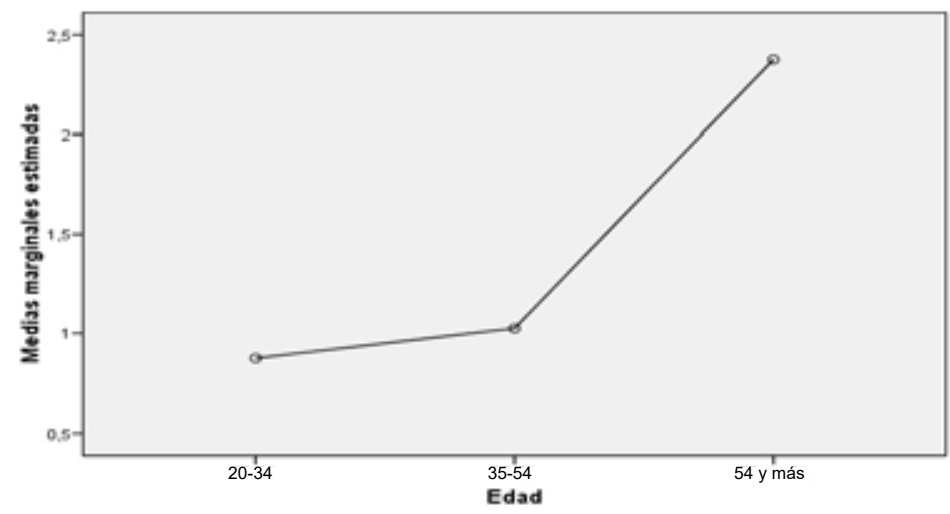

\subsubsection{Grupo socioeconómico}

En la tabla 6 y el gráfico 7, se muestran las frecuencias absoluta y porcentual de la ocurrencia de no dequeísmo y dequeísmo identificadas en el corpus, según el grupo socioeconómico de los sujetos.

Según nuestros datos, en términos estadísticos descriptivos, no se perciben diferencias notables entre los porcentajes de ocurrencia de las variantes analizadas correspondientes a cada grupo socioeconómico. La comparación de las medias correspondientes a la frecuencia del no dequeísmo, según el grupo socioeconómico de los sujetos, se expone en el gráfico 8. La prueba ANOVA proporciona los siguientes resultados: bajo (42,767), medio bajo $(57,233)$, medio $(71,533)$ y medio alto $(81,000)$, los que son significativos estadísticamente, puesto que $\mathrm{F}=10,983, \mathrm{p}=0,000$. En consecuencia, nuestro análisis sugiere que habría una relación entre el no dequeísmo y los sujetos del nivel socioeconómico medio alto. 
Tabla 6. Frecuencia absoluta y porcentaje de las variantes no dequeísta y dequeísta en el corpus según el grupo socioeconómico de los sujetos de la muestra

\begin{tabular}{lccc}
\hline Grupo & No dequeísmo & Dequeísmo & Totales \\
\hline Bajo & $1283(97,9)$ & $27(2,1)$ & $1310(100)$ \\
Medio Bajo & $1717(97,4)$ & $45(2,6)$ & $1762(100)$ \\
Medio & $2146(98)$ & $43(2)$ & $2189(100)$ \\
Medio alto & $2430(97,7)$ & $56(2,3)$ & $2486(100)$ \\
\hline
\end{tabular}

Gráfico 7. Porcentaje de frecuencia de las variantes no dequeísta y dequeísta en el corpus según el grupo socioeconómico de los sujetos de la muestra

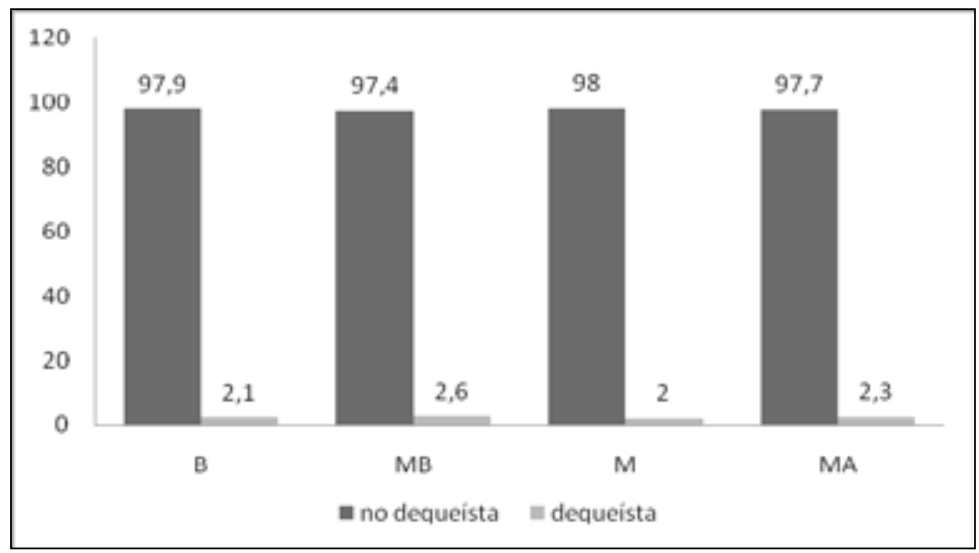

Los resultados del análisis paramétrico del dequeísmo, por su parte, son los siguientes: bajo $(0,900)$, medio bajo $(1,500)$, medio $(1,433)$ y medio alto $(1,867)$, si bien estas diferencias no son estadísticamente significativas $(\mathrm{F}=0,637, \mathrm{p}=0,592)$. A su vez, la prueba no paramétrica seleccionada entrega los siguientes rangos promedio: bajo $(51,55)$, medio bajo $(58,72)$, medio $(63,67)$ y medio alto $(68,07)$, confirmando que estas diferencias no son significativas, ya que Chicuadrado $=4,417, \mathrm{p}=0,220$. 


\section{Gráfico 8. Medias marginales estimadas de no dequeísta}

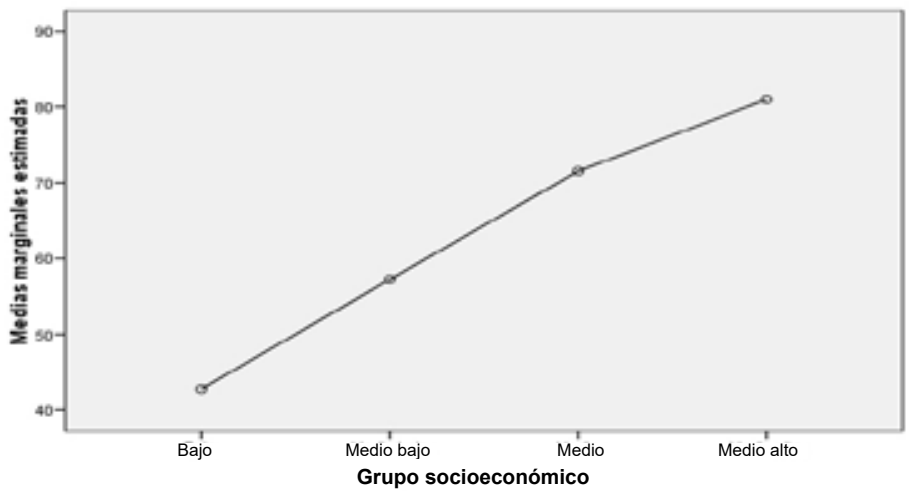

Mc Lauchlan(1982), Boretti de Macchia(1989), Carbonero(1991), Gómez Molina y Gómez Devís (1995), Serrano (1998) y Almeida (2009) coinciden con nuestros datos al destacar que el dequeísmo es más frecuente en los hombres; sin embargo, Bentivoglio (19801981), Del Valle Rodás (1996-1997) y Prieto (1995-1996), informan que lo es en las mujeres. Por otro lado, Bentivoglio (1980-1981), Prieto (1995-1996) y Serrano (1998) informan que el dequeísmo es característico en los sujetos de edad intermedia, mientras Boretti de Macchia (1989) y Del Valle Rodás (1996-1997), que lo es en hablantes jóvenes. Ninguno de los estudios revisados señala la posibilidad de un vínculo del dequeísmo con sujetos de edad avanzada como proponemos aquí. Finalmente, Bentivoglio (1980-1981), Gómez Molina y Gómez Devís (1995), Prieto (1995-1996) y Serrano (1998) señalan que el dequeísmo es más común en los niveles sociales medios.

\section{Conclusiones}

1. Se identificaron 7747 casos de alternancia de la variable "ausencia o presencia de la preposición de ante un que conjuntivo o relativo cuando es gramaticalmente innecesaria”, de los cuales 7576 (97,8\%) casos corresponden a su ausencia (o variante no dequeísta) y $171(2,2 \%)$, a su presencia (o variante dequeísta). En consecuencia, 
nuestro análisis mostró que la presencia de la preposición de ante un que conjuntivo o relativo es muy inferior a su ausencia, cuando es sintácticamente expletiva, en el corpus analizado. Este resultado también coincide con la mayor parte de los autores que han estudiado el dequeísmo, quienes destacan que está mucho menos difundido que el queísmo, en las comunidades de habla española ( $c f$. Almeida 2009). Nuestros datos evidencian un ligero avance del dequeísmo desde la década de 1990 hasta nuestros días, en consideración a los resultados de Prieto (1995-1996); esto nos conduce a suponer que se trata de un fenómeno más bien estable.

2. En cuanto a la incidencia de los factores lingüísticos, en términos de frecuencia relativa, el dequeísmo ocurre mayormente como sustitución de otras preposiciones y en estructuras apositivas (7,5\% cada uno). A continuación, está el dequeísmo en locuciones conjuntivas o adverbiales $(4,7 \%)$ y delante de un que relativo $(4 \%)$. Le sigue la adición de de ante un que conjuntivo en cláusulas con la función de objeto directo $(2 \%)$ y de sujeto $(1,8 \%)$. En cláusulas de atributo, el dequeísmo fue casi inexistente $(0,2 \%)$. No obstante, en términos de frecuencia absoluta, la mayor parte de los casos de dequeísmo en el corpus se dieron en cláusulas de objeto (89 ejemplos) y, en menor medida, de sujeto (32 ocurrencias). Específicamente, es muy frecuente la adición de la preposición de ante un que conjuntivo con verbos de cognición y enunciación y, en particular, con los verbos decir, ser, resultar, creer y pensar.

3. Por lo que se refiere a la determinación de los factores sociales, el dequeísmo se da más en hombres $(3,1 \%)$ que en mujeres $(1,3 \%)$, diferencias que resultaron ser significativas estadísticamente. Asimismo, con proximidad a la significación estadística, concluimos que los hablantes del grupo de edad 55 y más años $(3,8 \%)$ tienden más hacia el empleo del dequeísmo que los de los grupos etarios 20 a $34(1,3 \%)$ y 35 a 54 (1,6\%). El dequeísmo no mostró diferencias porcentuales importantes entre los diferentes grupos socioeconómicos bajo (2,1\%), medio bajo (2,6\%), medio (2\%) y medio alto $(2,3 \%)$, ni tampoco este factor resultó ser significativo estadísticamente. 
4. En consecuencia, de acuerdo con estos datos, pudimos comprobar nuestra hipótesis sobre el influjo de los factores internos y externos en la ocurrencia del dequeísmo.

5. A nuestro juicio, tal vez, podría concluirse que el dequeísmo no tiene un prestigio abierto en Santiago, debido a que es más frecuente en los hombres, quienes son más proclives al empleo de rasgos lingüísticos característicos del prestigio encubierto ( $c f$. López Morales 2004, Moreno Fernández 2009, Trudgill y Campoy 2007: s. v. prestigio). Las ocurrencias del dequeísmo podrían deberse al desconocimiento de las reglas que rigen el uso preposicional, el que estaría motivado por el reducido espacio asignado a la enseñanza de la gramática en el currículo escolar actual. Asimismo, al parecer, el dequeísmo aún conserva la estigmatización señalada por Rabanales (1974) en la década de los setenta, si bien se emplea también entre hablantes del grupo medio alto. Esto último no debería extrañarnos, ya que el desconocimiento de este aspecto de la gramática española podría ser transversal. Su bajísima propagación en algunos dialectos del español podría explicarse por el hecho de que consiste en un proceso de inserción y, por lo tanto, podría interpretárselo como una solución menos económica que la elisión de la preposición. Para esta interpretación también encontramos respaldo en lo señalado por Almeida (2009) sobre que su carácter menos económico motiva su empleo minoritario, en contraste con el queísmo.

\section{Referencias bibliográficas}

Almeida, Manuel

2009 "(De)queísmo y variación sociolingüística en una comunidad urbana canaria”. Revista de Filología. 27, 9-30.

ArJona, Marina

1978 "Anomalías en el uso de la preposición de en el español de México”. Anuario de Letras. XVII, 67-90.

1979 "Usos anómalos de la preposición de en el habla popular mexicana”. Anuario de Letras. XVI, 167-184. 
Bentivoglio, Paola

1976 "Queísmo y dequeísmo en el habla culta de Caracas". En Colloquium on Hispanic Linguistics. Eds., Frances Aid, Melvyn Resnick y Bohdan Saciuk. Washington: Georgetown University Press, 1-18.

1980-1981 “El dequeísmo en Venezuela: ¿un caso de ultracorrección?” Boletín de Filología. XXXI, 715-719.

Bentivoglio, Paola y Francesco D’Introno

1977 "Análisis sociolingüístico del dequeísmo en el habla de Caracas”. Boletín de la Academia Puertorriqueña de la Lengua Española. 6, 1, 58-82.

Blas Arroyo, José Luis

2005 Sociolingüistica del español. Desarrollos y perspectivas en el estudio de la lengua española en contexto social. Madrid: Cátedra.

Boretti de Macchia, Susana

1989 “(De)queísmo en el habla culta de Rosario”. Anuario de Lingüistica Hispánica. 5, 27-48.

Caravedo, Rocío

2003 "Problemas conceptuales y metodológicos de la lingüística de la variación". En Lengua, variación y contexto. Estudios dedicados a Humberto López Morales, II. Ed., Francisco Moreno Fernández, José Antonio Samper Padilla, María Vaquero, María Luz Gutiérrez Araus, César Hernández Alonso y Francisco Gimeno Menéndez. Madrid: Arco Libros, 541-557.

Carbonero, Pedro

1992 "Queísmo y dequeísmo en el habla culta de Sevilla: análisis contrastado con otras hablas peninsulares y americanas". En Scripta philologica in honorem Juan M. Lope Blanch, II. Ed., Elizabeth Luna Traill. México D. F.: Universidad Nacional Autónoma de México, 43-63.

Coseriu, Eugenio

1982 “Sistema, norma y habla”. En Teoría del lenguaje y lingüistica general. Madrid: Gredos, 11-113. 
De Mello, George

1995 "El dequeísmo en el español hablado contemporáneo: ¿Un caso de independencia semántica?” Hispanic Linguistics. 6, 7, 117-151.

Del Valle Rodás, Juana

1996-1997 "Para una lingüística interpretativa: (de)queísmo en el habla de Salta”. Anuario de Lingüistica Hispánica. XII, 797-818.

GARCÍA, Erica

1986 "El fenómeno (de)queísmo desde una perspectiva dinámica del uso de la lengua”. En Actas del II Congreso Internacional sobre El español de América. Ed., José Moreno de Alba. México D. F.: Universidad Nacional Autónoma de México, 46-65.

Gómez Molina, José y María Begoña Gómez Devís

1995 "Dequeísmo y queísmo en español hablado de Valencia: Factores lingüísticos y sociales". Anuario de Lingüistica Hispánica. XI, 193-220.

GÓmEz TORRego, Leonardo

1999 "La variación en las subordinadas sustantivas: dequeísmo y queísmo". En Gramática descriptiva de la lengua española. 2. Las construcciones sintácticas fundamentales. Relaciones temporales, aspectuales y modales. Eds., Ignacio Bosque y Violeta Demonte. Madrid: Espasa, 2105-2148.

Guirado, Kristel

2006 "Deixis proposicional en el habla de Caracas: Un análisis cuantitativo del (de)queísmo”. Boletín de Lingüistica. XVIII, 130-156.

Hernández Campoy, Juan Manuel y Manuel Almeida

2005 Metodología de la investigación sociolingüística. Málaga: Editorial Comares.

LABOv, William

1966 The social stratification of English in New York City. Washington: Center of Applied Linguistics.

1983 Modelos sociolingüisticos. Madrid: Cátedra.

2001 Principles of Linguistic Change. Vol. 2. Social Factors. Oxford: Blackwell. 
2004 "Quantitative reasoning in linguistics". En Sociolinguistics/ Soziolinguistik: An international handbook of the science of language and society. Vol I. Eds., Ulrich Ammon, Norbert Dittmar, Klaus J. Mattheier y Peter Trudgill. Berlín: Mouton de Gruyter, 6-22.

LAVANDERA, Beatriz

1978 "Where does the sociolinguistic variable stop?". Language in Society. 7, 171-182.

López Morales, Humberto

1994 Métodos de investigación lingüística. Salamanca: Ediciones Colegio de España.

2004 Sociolingüistica. Madrid: Gredos.

2008 "El estudio de la variación lingüística". En Estudios de lengua española: descripción, variación y uso. Homenaje a Humberto López Morales. Eds., María Victoria Camacho, José Rodríguez y Juana Santana. Madrid/Frankfurt: Iberoamericana/Vervuert, 7-34.

Mc Lauchlan, Jessica

1982 “Dequeísmo y queísmo en habla culta de Lima”. Lexis. 6, 1, 11-54.

Moreno Fernández, Francisco

1990 Metodología sociolingüística. Madrid: Editorial Gredos.

2009 Principios de sociolingüística y sociología del lenguaje. Barcelona: Ariel.

Prieto, Luis

1995-1996 "Análisis sociolingüístico del dequeísmo en el habla de Santiago de Chile”. Boletín de Filología. XXXV, 379-452.

Quilis SANZ, María José

1986 "El dequeísmo en el habla de Madrid y en la telerradiodifusión española”. Boletín de la Academia Puertorriqueña de la Lengua Española. XIV, 139-150.

Rabanales, Ambrosio

1974 “Queísmo y dequeísmo en el español de Chile”. En Estudios filológicos y lingüísticos. Homenaje a Ángel Rosenblat. Ed., María Josefina Tejera. Caracas: Instituto Pedagógico, 413-444. Métodos probatorios en gramática científica. Madrid: Istmo. 
RAE (Real Academia Española)

1973 Esbozo de una nueva gramática de la lengua española. Madrid: Espasa Calpe.

2005 Diccionario panbispánico de dudas. Madrid: Espasa.

2011 Nueva gramática básica de la lengua española. Barcelona: Espasa.

Romaine, Suzanne

1996 El lenguaje en la sociedad. Barcelona: Ariel.

San Martín, Abelardo y Silvana Guerrero

2015 "Estudio Sociolingüístico del Español de Chile (ESECH): recogida y estratificación del corpus de Santiago”. Boletín de Filología. L, 1, 221-247. https://doi.org/10.4067/s071893032015000100009

SCHWENTER, Scott

1999 "Evidentiality in Spanish morphosyntax: a reanalysis of (de) queísmo". En Estudios de variación sintáctica. Ed., María José Serrano. Madrid/Frankfurt: Iberoamericana/Vervuert, 65-87.

SeCo, Manuel

1989 Diccionario de dudas y dificultades de la lengua española. Madrid: Espasa Calpe.

Serrano, María José

1998 "Estudio sociolingüístico de una variante sintáctica: el fenómeno dequeísmo en español canario”. Hispania. 81, 392-405. https://doi.org/10.2307/345054

2011 Sociolingüística. Barcelona: Ediciones del Serbal. https://doi. org/10.2307/345054

Silva-Corvalán, Carmen

2001 Sociolingüística y pragmática del español. Washington: Georgetown University Press.

Trudgill, Peter y Juan Manuel Hernández Campoy

2007 Diccionario de sociolingüística. Madrid: Gredos.

Recibido: 09/11/2015

Aceptado: 06/02/2017 